\title{
Chapter 25 \\ Blood Pressure and the Risk of Acute Kidney Injury in the ICU: Case-Control Versus Case-Crossover Designs
}

\author{
Li-wei H. Lehman, Mengling Feng, Yijun Yang and Roger G. Mark
}

\section{Learning Objectives}

Introduce two different approaches, a case-control and a case-crossover design, to study the effect of transient exposure of hypotension on the risk of acute kidney injury (AKI) development in intensive care unit (ICU) patients.

\subsection{Introduction}

Acute kidney injury (AKI) refers to a rapid decrease in kidney function, occurring over a period of days. The presence of AKI can be detected using well-established definitions based on serum creatinine rise or urine output reduction [1]. Acute kidney injury has been reported in $36 \%$ of all patients admitted to the intensive care unit ICU $[2,3]$. A prior study showed that hospital patients with even very small increases in their serum creatinine $(0.3-0.4 \mathrm{mg} / \mathrm{dL})$ have $70 \%$ greater risk of death than patients without creatinine increase [4]. Although the relationship between low blood pressure and kidney function is well documented in an experimental setting based on animal data [5], the association between hypotension and acute kidney injury in a critical care setting is not completely understood.

This chapter describes two different approaches for studying blood pressure and the risk of AKI development in ICU patients using the MIMIC II database [6]. In our first study, we adopted a traditional case-control approach and examined the association between hypotension and AKI by comparing blood pressure measurements of patients who had AKI (case) with patients without AKI (control) [7, 8]. Blood pressure measurements immediately prior to patients' AKI onset were compared with blood pressure measurements of the controls sampled from a similar time window.

In the second study, we adopted a case-crossover design in which each patient serves as his or her own control. Blood pressure measurements immediately prior to each patient's AKI onset were compared with the same patient's blood pressure 
measurements sampled from an earlier time window while that patient's kidney functions were still stable. In the remainder of the chapter, we highlight the key differences and the design rationale of these two approaches. We applied these analysis techniques to study the relationship between hypotension and AKI development using the MIMIC II database, and present our preliminary findings.

\subsection{Methods}

\subsubsection{Data Pre-processing}

Nurse-verified mean arterial blood pressure (MAP) samples, recorded on an hourly basis were used for the analysis. Blood pressure measurements from both invasive arterial line and automated, non-invasive oscillometric methods were included in the study. Our choice of MAP (rather than systolic blood pressure) for blood pressure measurement was motivated by prior work [8] which demonstrated that MAP provided more consistent readings across different measurement modalities in the ICU. Blood pressure measurements were filtered to remove values outside of reasonable physiological bounds (MAP between 20 and $200 \mathrm{mmHg}$ ).

\subsubsection{A Case-Control Study}

In the case-control approach [7], we examined the effect of transient exposure to hypotension (defined as blood pressure falling below specified thresholds) and the risk of AKI development by comparing blood pressure measurements of patients who experienced AKI (case) with patients who never developed AKI in the ICU (control). AKI was defined as an acute increase in serum creatinine $\geq 0.3 \mathrm{mg} / \mathrm{dL}$, or an increase of $\geq 50 \%$ in serum creatinine within $48 \mathrm{~h}$, based on the Acute Kidney Injury Network (AKIN) definition [1]. Blood pressure measurements (from up to a $48 \mathrm{~h}$ window) prior to patients' AKI onset were compared with blood pressure measurements of the controls from a time window prior to the last creatinine measurement time.

Patients were selected from among the adult ICU stays in the MIMIC II [8] database. We examined adult ICU stays (patients $\geq 15$ years of age) with at least 2 serum creatinine values. Patients with fewer than 2 serum creatine values in their ICU stay or evidence of end-stage renal disease (ESRD) were excluded.

Among the remaining 16,728 adult ICU stays that had at least 2 creatinine measurements without evidence of end-stage renal disease, AKI occurred in 5207 (31\%). The remaining 11,521 cases were identified as the controls. The average AKI onset time was 2.34 days after ICU admission. For the controls, the last creatinine sample time was, on average, 2.76 days after ICU admission. Figure 25.1 


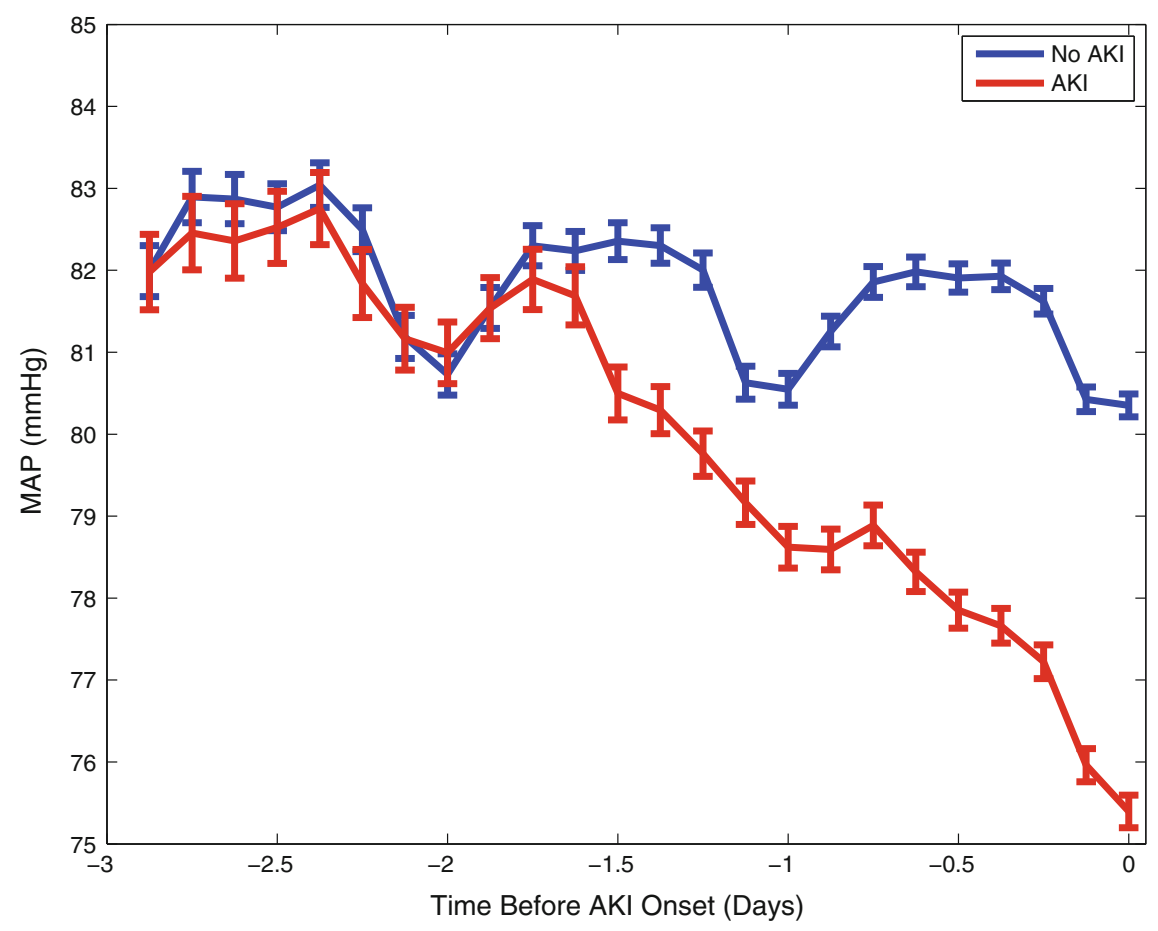

Fig. 25.1 The population mean (and standard error) of median MAP up to 3 days prior to the AKI onset for the AKI cohort, or prior to the last creatinine measurement time for the controls. Mean arterial blood pressure of the AKI cohort diverged from that of the controls during day two prior to the AKI onset, and both cohorts exhibited prominent diurnal variation

plots the population mean and standard error of median MAP up to 3 days prior to the AKI onset for the AKI cohort, or prior to the last creatinine measurement time for the controls. Note that mean arterial blood pressure of the AKI cohort diverged from that of the controls prior to the AKI onset.

We studied the risk of AKI in ICU patients as a function of both the severity and duration of hypotension. Blood pressure features extracted from the target 48-h window were examined as primary predictors for AKI, including the minimum MAP and maximum number of hours that MAP was continuously less than several different thresholds (from 80 to $45 \mathrm{mmHg}$ ). Duration of hypotension below a specific threshold was calculated based on linear interpolated blood pressure samples. Hypotensive episodes were considered to begin and end when the interpolated blood pressure values intercepted the target threshold. Hypotensive episodes that were less than one hour apart were merged to form one continuous episode.

Univariate and multivariable logistic regressions were performed to find correlations between hypotension and AKI. Age, SAPS-I, admission creatinine, and the 
presence (based on ICD-9) of chronic renal failure (585.9), hypertension (401.9), diabetes (250.00), coronary atherosclerosis (414.01), congestive heart failure (428.0), and septic shock (785.52) or sepsis (038) were added as potential confounding factors [9].

Our results indicate that the odds of AKI were related to the severity of hypotension with an odds ratio (OR) of 1.03, $95 \%$ confidence interval (CI) 1.02$1.04(p<0.0001)$ per $1 \mathrm{mmHg}$ decrease in minimum MAP $\leq 80 \mathrm{mmHg}$. Multivariable analysis on hypotension duration involved 3203 patients who had SAPS-I scores and with at least $45 \mathrm{~h}$ of blood pressure samples in the target $48-\mathrm{h}$ window. Our results indicate that the duration of time that the patient's MAP was continuously less than or equal to $70,65,60,55$, and $50 \mathrm{mmHg}$ were significant risk factors in AKI development. Further, as the extent of hypotension worsened, the incremental risk for AKI from each additional hour of continuous hypotension increased for each $10 \mathrm{mmHg}$ drop in MAP below $80 \mathrm{mmHg}$. For each additional hour MAP was less than 70,60, $50 \mathrm{mmHg}$, the odds of AKI increased by $2 \%$ (OR $1.02,95 \%$ CI $1.00-1.03, p=0.0034), 5 \%$ (OR 1.05, $95 \%$ CI 1.02-1.08, $p=0.0028)$, and $22 \%$ (OR 1.22, $95 \%$ CI 1.04-1.43, $p=0.0122)$ respectively. As the degree of hypotension worsened, the increased odds for AKI from each additional hour of continuous hypotension more than doubled for each $10 \mathrm{mmHg}$ drop in MAP below $80 \mathrm{mmHg}$. Our results also suggest that the severity of hypotension significantly shortened the time to the onset of AKI.

\subsubsection{A Case-Crossover Design}

In the second study, we adopted a case-crossover cohort design to examine the effect of transient exposure to hypotension and the risk of AKI. The case-crossover design was devised to assess the relationship between transient exposures and acute outcomes in situations where the control series of a case-control study is difficult to achieve. In the case-crossover design, subjects serve as their own matched controls defined by prior time periods in the same subject. Given a transient exposure with stable prevalence over time, the case-crossover design uses the difference in exposure rates just before an event (case) with those at other time points in the subject's history (controls) to estimate an odds ratio of the outcome associated with exposure. The case-crossover design was first proposed by Maclure et al. to study the effects of transient changes on the risk of acute events [10]. One advantage of a case-crossover design is that it avoids control selection bias and eliminates between-patient confounding factors $[10,11]$. In this study design, the AKI definition is based on hourly urine output (instead of daily creatinine measurements) in order to determine a more precise timing of the acute (oliguria) onset.

Adult patients with normal kidney function (i.e. urine output remaining at $0.5 \mathrm{ml} / \mathrm{kg} / \mathrm{h}$ or above) during the first $12 \mathrm{~h}$ in the ICU, who subsequently developed 
AKI/oliguria (urine output remains below $0.5 \mathrm{ml} / \mathrm{kg} / \mathrm{h}$ for at least $6 \mathrm{~h}$ ) in the ICU were included in the study. The same patients, prior to developing AKI/oliguria, were used as controls. The AKI/oliguria onset was defined as the beginning of the 6-h period when urine output remained below $0.5 \mathrm{ml} / \mathrm{kg} / \mathrm{h}$.

The minimum MAP from the $3 \mathrm{~h}$ period prior to the AKI onset was used as exposure for the cases. The minimum MAP from a 3-h control period during the first $12 \mathrm{~h}$ in the ICU, when the same patient's renal function was still normal, was used as exposure for the controls. Since the blood pressure measurements during the first $6 \mathrm{~h}$ patients were in the ICU can be sparse, we chose the control period to be the 7th-9th hour from the beginning of the patients' ICU stays. Blood pressure measurements were filtered to remove outliers as before.

Case-crossover designs are typically analyzed using conditional logistic regression, as it accounts for the matched nature of the data. It is analogous to a matched case-control study, where one compares a 'case' person-moment with a series of 'control' person-moments from different subjects, while in the case-crossover design, the 'control' person-moments are from the same subject. We implemented the latter approach for analyzing case-crossover study data. In addition, time-varying confounding factors (mechanical ventilator, vasopressors, temperature, heart rate, white blood cell count, $\mathrm{SpO}_{2}$ ) were included in the multivariable conditional logistic regression model.

The total cohort included 911 adult ICU stays (29.86\% MICU, $21.73 \%$ SICU, $22.94 \% \mathrm{CCU}, 25.47 \% \mathrm{CSRU})$ from the MIMIC II database. The median time to AKI/oliguria onset was $45 \mathrm{~h}$. The population median of the minimum MAP measurements during the control and case periods were $73 \mathrm{mmHg}$ with an inter-quartile range of $[65,83] \mathrm{mmHg}$, and $70[62,79] \mathrm{mmHg}$ respectively. A paired signed T-test indicates that the minimum MAP during the case period is statistically significantly lower than during the control period $(p$-value $=0.0001)$. Our results indicate that the odds of AKI were related to the severity of hypotension with an odds ratio (OR) of 1.035, $95 \%$ confidence interval (CI) 1.024-1.045 $(p<0.0001)$ per $1 \mathrm{mmHg}$ decrease in minimum MAP in multivariable conditional logistic regression after adjusting for temperature, heart rate, $\mathrm{SpO}_{2}$, white blood cell count, and the use of mechanical ventilation and vasopressors. Furthermore, we performed a similar analysis to understand if the risk of developing AKI increases associated with the worsened hypotension treating the minimum MAP at the binary variable using cutoff of 70, 65, 60, 55, and $50 \mathrm{mmHg}$. The adjusted odds ratios and $95 \% \mathrm{CI}$ for the minimum MAP $<70$, MAP $<65$, MAP $<60$, MAP $<55$, and MAP $<50$ (vs. when MAP was greater than or equal to the respective thresholds) were 1.854 (1.44-2.38), 1.945 (1.502-2.519), 2.096 (1.532-2.869), 2.002 (1.307-3.065), and 2.107 (1.115-3.982), respectively. These findings are consistent with the results described in the previous section using a case-control study design. 


\subsection{Discussion}

In the study of the association of hypotension with AKI, the case-crossover design is an efficient alternative to the case-control approach. The case-crossover design, based exclusively on the case series, performs within-subject comparisons of blood pressure measurements from the case and the control periods to estimate the rate ratio of the AKI outcome associated with hypotension. This design inherently removes the biasing effects of unmeasured, time-invariant confounding factors from the estimated rate ratio.

Many factors, (including chronic kidney disease, hypertension, diabetes) could potentially contribute to the development of AKI in an ICU setting. In a traditional case-control design, these time-invariant between-patient confounders (as well as the time-varying confounders) would have to be included to adjust for the baseline risk of AKI development. In some cases, these confounding variables can be difficult to determine from a retrospective ICU database. In a case-crossover design, each patient's blood pressure during normal renal function is compared with the same patient's blood pressure immediately prior to AKI onset, so that time-invariant patient characteristics and confounders are eliminated in the analysis. A case-crossover design may be a more efficient approach in investigating the transient effect of exposure (e.g. low blood pressure) on the risk of an acute outcome (e.g. AKI development), when the heterogeneity in the baseline risk may be difficult to account for in the conventional case-control design.

We acknowledge the following limitations in the current study. First, this was a retrospective study, and as such, the incidence of hypotension prior to AKI does not prove a causal mechanism. Second, we did not account for the presence of fluid and several interventions (e.g. contrast agents, NSAIDs, aminoglycosides, ACEI, etc.) that may impair renal function in our multivariable analysis. As part of future work, additional time-varying confounders (such as, usage of Lasix within $6 \mathrm{~h}$, IV fluid, creatinine, time of AKI onset) could be included in the model.

\subsection{Conclusions}

We have presented two different approaches, a case-control and a case-crossover design, to study the effect of transient exposure to hypotension on the risk of AKI development in ICU patients. Results from multivariable analysis in both studies indicate that hypotension is a statistically significant risk factor in the development of AKI in the ICU. This study serves as an example to illustrate the utility of case-crossover designs to study the association between a risk factor and the subsequent disease development in an EHR-based retrospective clinical analysis.

Open Access This chapter is distributed under the terms of the Creative Commons Attribution-NonCommercial 4.0 International License (http://creativecommons.org/licenses/by-nc/ $4.0 /$ ), which permits any noncommercial use, duplication, adaptation, distribution and reproduction 
in any medium or format, as long as you give appropriate credit to the original author(s) and the source, a link is provided to the Creative Commons license and any changes made are indicated.

The images or other third party material in this chapter are included in the work's Creative Commons license, unless indicated otherwise in the credit line; if such material is not included in the work's Creative Commons license and the respective action is not permitted by statutory regulation, users will need to obtain permission from the license holder to duplicate, adapt or reproduce the material.

\section{Code Appendix}

The code used in this case study is available from the GitHub repository accompanying this book: https://github.com/MIT-LCP/critical-data-book. Further information on the code is available from this website.

\section{References}

1. Mehta RL, Kellum JA, Shah SV, Molitoris BA, Ronco C, Warnock DG, Levin A (2007) Acute kidney injury network (AKIN): report of an initiative to improve outcomes in acute kidney injury. Crit Care 11:R31

2. Bagshaw S, George C, Dinu I, Bellomo R (2008) A multi-center evaluation of the RIFLE criteria for early acute kidney injury in critically ill patients. Nephrol Dial Transplant 23:1203-1210

3. Ostermann M, Chang R (2007) Acute kidney injury in the intensive care unit according to rifle. Crit Care Med 35:1837-1843

4. Chertow G, Burdick E, Honour M, Bonventre J, Bates D (2005) Acute kidney injury, mortality, length of stay, and costs in hospitalized patients. J Am Soc Nephrol 16:3365-3370

5. Kirchheim HR, Ehmke H, Hackenthal E, Löwe W, Persson P (1987) Autoregulation of renal blood flow, glomerular filtration rate and renin release in conscious dogs. Pflugers Archiv Eur J Physiol 410:441-449

6. Saeed M, Villarroel M, Reisner AT, Clifford G, Lehman LH, Moody G, Heldt T, Kyaw TH, Moody B, Mark RG (2011) Multiparameter intelligent monitoring in intensive care (MIMIC II): a public-access intensive care unit database. Crit Care Med (5):952-960

7. Lehman LH, Saeed M, Moody G, Mark R (2010) Hypotension as a risk factor for acute kidney injury in ICU patients. In: Computing in cardiology 2010. IEEE Computer Society Press, Belfast, pp 1095-1098

8. Lehman LH, Saeed M, Talmor D, Mark RG, Malhotra A (2013) Methods of blood pressure measurement in the ICU. Crit Care Med 41(1):3-40

9. Abuelo G (2007) Normotensive ischemic acute renal failure. N Engl J Med 357:797-805

10. Maclure M (1991) The case-crossover design: a method for studying transient effects on the risk of acute events. Am J Epidemiol 133:144-153

11. Maclure M, Mittleman M (2000) Should we use a case-crossover design. Annu Rev Public Health 21:193-221 